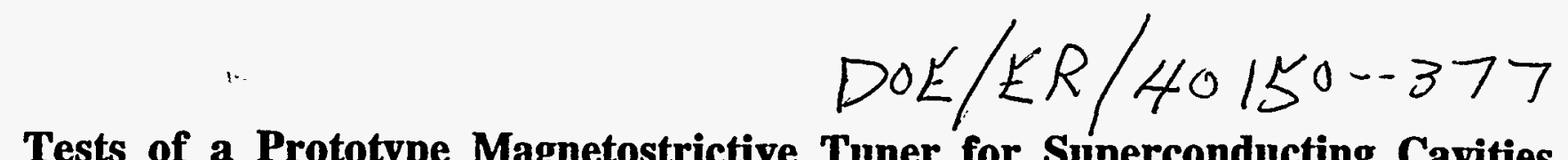

\title{
Tests of a Prototype Magnetostrictive Tuner for Superconducting Cavities
}

\author{
J. F. Benesch and M. Wiseman* \\ Thomas Jefferson National Accelerator Facility, Newport News, VA 23606 USA E CEIVED
}

\begin{abstract}
The Continuous Electron Beam Accelerator (CEBA) uses mechanical tuners at $2 \mathrm{~K}$ driven by room temperature stepping motors in a feedback loop to maintain cavity frequency at 1497 MHz. A modification of the existing system was designed, replacing a passive section of the mechanical tuner with a magnetostrictive tuning element consisting of a nickel rod and an Industrially supplied $0.25 \mathrm{~T}$ superconducting solenoid. This assembly was tested with several magnetic shield configurations designed to keep the stray nux at the niobium cavity below $1 \mu T$ when the cavity was normal, to maintain cavity $Q$. Results of the tests, including change in cavity performance when the cavity was locally quenched near the end of the solenold, showed that a multilayer shield of $6 \mathrm{~mm}$ of steel, with sheets of $\mathrm{mu}$ metal, niobium and mu metal spaced appropriately outside the thick steel, was effective in containing the Iux, both remanent and current-driven, preventing any change in cavity $Q$ upon cooldown or quench with an external heater near the end of the solenoid. Hysteresis attributed to the nickel magnetostrictive element was observed. *This work was supported by U.S. Department of Energy Contract DE-AC05-84ER40150.
\end{abstract}

\section{INTRODUCTION}

The momentum and emittance specifications of the CEBA $^{1}$ required the design of a mechanical tuning system with wide range and good accuracy which could be controlled by a feedback loop to keep the resonant frequency of each of 338 cavities within $\pm 25 \mathrm{~Hz}$ of a master oscillator signal at $1497 \mathrm{MHz}$. When the cavity frequency strays outside this band, the feedback system activates a stepper motor to bring the cavity back within $\pm 5 \mathrm{~Hz}$. This system is complicated by the backlash associated with the mechanical gear train, the slow response of the mechanical system, and the finite step size.

A system with one second response is desired for CEBA performance improvement and is necessary for the Thomas Jefferson National Accelerator Facility (Jefferson Lab, formerly known as CEBAF) prototype IR FEL ${ }^{2}$. This system will be used in a feedback loop inside that goveming the mechanical system, so the frequency adjustment capability of the new system need be only $500 \mathrm{~Hz}$. The mechanical system has a $1 \mathrm{MHz}$ range. After an informal review of the piezoelectric and magnetostrictive systems which have been used at other laboratories, it was decided to modify the existing mechanical tuner design by incorporating a magnetostrictive element (fig. 1). None of the modern magnetostrictive materials had been applied at $2 \mathrm{~K}$, and data was available for nickel at $4 \mathrm{~K}$, so that was chosen. A peak field of $0.25 \mathrm{~T}$ was targeted, encompassing $\sim 75 \%$ of the available length change in nickel. ${ }^{3}$

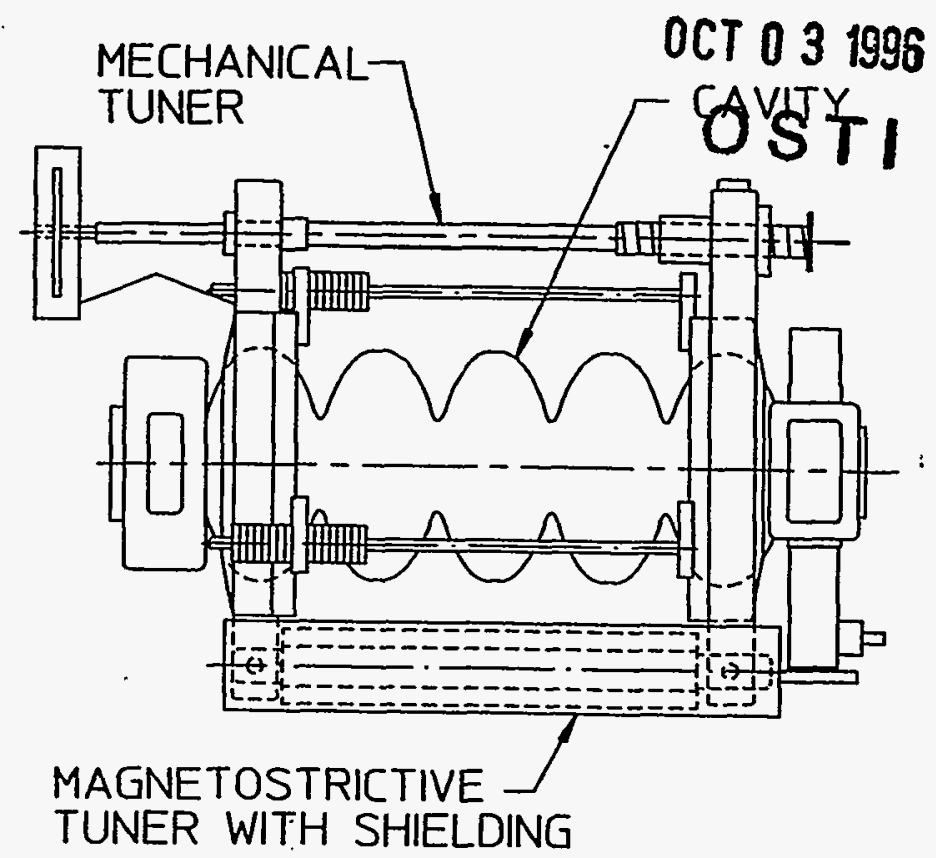

Fig. 1 Tuner configuration

Since magnetostriction produces only a length reduction, control in both directions about $1497 \mathrm{MHz}$ is to be supplied by tuning the cavity within a few $\mathrm{KHz}$. mechanically, biasing the magnetostrictive tuner to the middle of its range, and then using the mechanical tuner to set the frequency at $1497 \mathrm{MHz} \pm 5 \mathrm{~Hz}$. Altering the current to the solenoid will then provide positive and negative range about the set point. The software for the mechanical system will bring it into play to bring the solenoid current back to nominal when the magnetostrictive tuner reaches its limits.

\section{DISCUSSION}

A specification for a simple superconducting solenoid with iron magnetic shield and field values in the bore and outside the shield was. written and sent to three vendors. The contract was awarded to Cryomagnetics, Inc., and design proceeded interactively with W. McGhee of that firm. The final design had bore field of $0.24 \mathrm{~T}$ at $10 \mathrm{~A}$ and stray field of $400 \mu \mathrm{T}$ at $\mathrm{R}=0, \mathrm{Z}=0.24 \mathrm{~m}$ and $270 \mu \mathrm{T}$ maximum at the cavity surface. Bore field was measured at the vendor; external field was not. It was agreed that supplemental, external shields would be designed at Jefferson Lab. For reasons of cost, the steel shield was fabricated from a common steel pipe.

Magnetic field at the surface of the niobium cavities used in CEBA must be maintained below $1 \mu \mathrm{T}$ to obtain the desired $Q=10^{10}$ at $2 \mathrm{~K}$. Since both the nickel magnetostrictive element and the steel shield are relatively 


\section{DISCLAIMER}

Portions of this document may be illegible in electronic image products. Images are produced from the best available original document. 


\section{DISCLAIMER}

This report was prepared as an account of work sponsored by an agency of the United States Government. Neither the United States Government nor any agency thereof, nor any of their employees, makes any warranty, express or implied, or assumes any legal liability or responsibility for the accuracy, completeness, or usefulness of any information, apparatus, product, or process disclosed, or represents that its use would not infringe privately owned rights. Reference herein to any specific commercial product, process, or service by trade name, trademark, manufacturer, or otherwise does not necessarily constitute or imply its endorsement, recommendation, or favoring by the United States Government or any agency thereof. The views and opinions of authors expressed herein do not necessarily state or reflect those of the United States Government or any agency thereof. 


\section{RECEIVED \\ OCT 031996 \\ OSTI}

This report has been reproduced from the best available copy.

Available to DOE and DOE contractors from the Office of Scientific and Technical Information, P.O. Box 62, Oak Ridge, TN.37831; prices available from (615)576-8401, FTS 626-8401.

Available to the public from the National Technical Information Service, U.S. Department of Commerce, 5285 Port Royal Rd., Springfield, VA 22161.

Price: Printed Copy $A O 2$

Microfiche A01 
hard magnetically, remanent field was a concern. The upllemental shield was designed empirically, because the mechanical attachments to the cavity required complex gaps and we wouldn't have believed the 3D calculations to the required field level in any event. Two layers of $250 \mu \mathrm{m} \mathrm{mu}$ metal were used outside the steel, with spacing of $6 \mathrm{~mm}$, to contain the remanent field while the system was above the critical temperature of niobium. Below that temperature, the cavity self-shielded - unless it quenched.

Superconducting cavities quench due to a material defect or heating due to field emission from a surface feature. The quenches are typically localized. It is thus necessary to shield the cavity from the coil's flux even when the system is at $2 \mathrm{~K}$, because stray flux will penetrate the cavity and remain there, lowering $\mathbf{Q}$, upon recovery. Thus a more substantial magnetic shield, one sufficient to contain $99+\%$ of the return flux of the solenoid, was also needed. Two types were tested, the vendor supplied iron and a Jefferson Lab-fabricated niobium sheet shield, also $250 \mu \mathrm{m}$.

Four configurations were tested:

1. Cavity without tuner, to get a baseline with no local magnetic material

2. Cavity/tuner with iron shield and two layers of mu metal 3. Cavity/tuner with niobium shield and two layers of $\mathrm{mu}$ metal

4. Cavity/tuner with iron shield, mu metal, niobium and mu metal, in that order.

Only in the last configuration was there no change in cavity $Q$ when the cavity was quenched with the solenoid energized at $150 \%$ of design current. Configurations 2 and 3 showed $Q$ reduction by a factor of three after a local quench was induced near the end of the solenoid.

The system was also tested with a $2 \mathrm{~A}$ sine wave of $10 \mathrm{~Hz}$ in the solenoid, and appeared to track, but it is difficult to measure frequencies to an accuracy of a few $\mathrm{Hz}$ if they are changing at a $10 \mathrm{~Hz}$ rate.

Figure 2 shows the frequency of the cavity versus solenoid current in test configuration 4. The data was manually logged using a 0-10 A, manually controlled power supply. The center frequency in these tests is not set to 1497 MHz because the vertical dewar does not have provisions for connection of a stepper motor to the mechanical tuner. Note that the frequency response fits very well with the log of the solenoid current. While not understood, this simple function will make the software easier to write. The fact that the middle of the range is $\sim 1 \mathrm{~A}$ will reduce resistive heating via the leads to the $2 \mathrm{~K}$ bath. The leads are connected thermally to the $60 \mathrm{~K}$ shield in the cryostat.

Figure 3 shows two cycles through the current range $0.75-1.5 \mathrm{~A}$. Frequency shift is $-60 \mathrm{~Hz}$ and width of the hysteresis loop is $\sim 10 \mathrm{~Hz}$. This hysteresis persisted at this amplitude even in test 3 , when the only non-linear elements were the nickel magnetostrictive rod and the two mu metal shields which would have saturated at much lower field. It is concluded that the hysteresis is due to the magnetostrictive element, and cannot be avoided. The iron shield contributes less than $1 \%$ of the bore field, and so was not expected to be a cause of hysteresis. This was confirmed by test 3 . The iron shield was vacuum annealed after several tests to "soften" its magnetic properties; this did not change performance either.

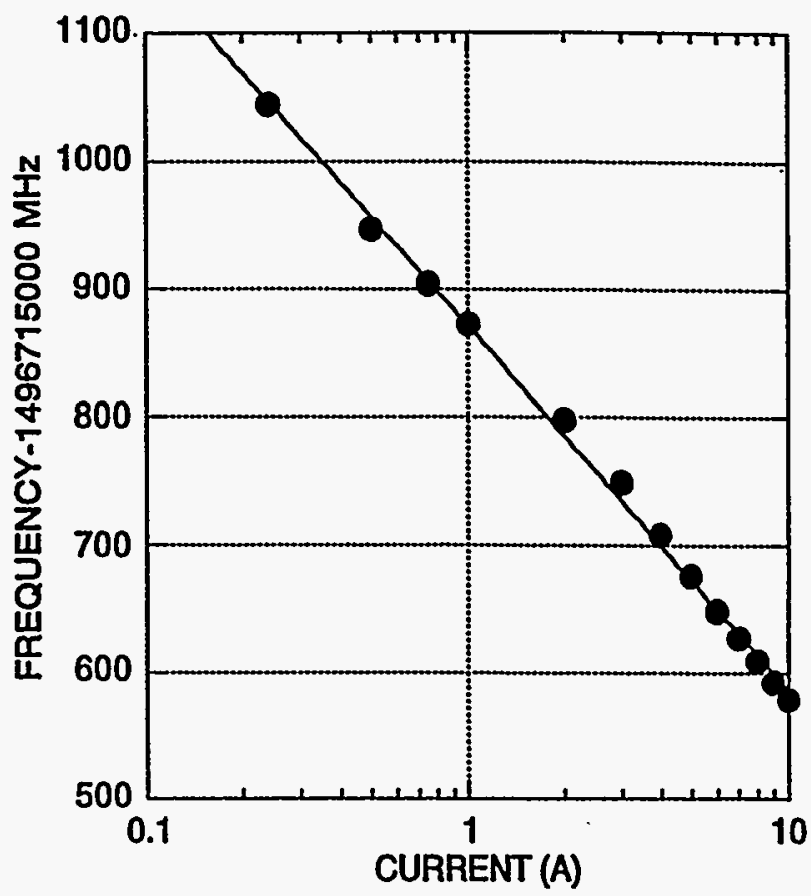

Fig. 2. Frequency change as a function of solenoid current, $0.2-10 \mathrm{~A}$, showing logarithmic response and $500+\mathrm{Hz}$ range available from the magnetostrictive tuner.

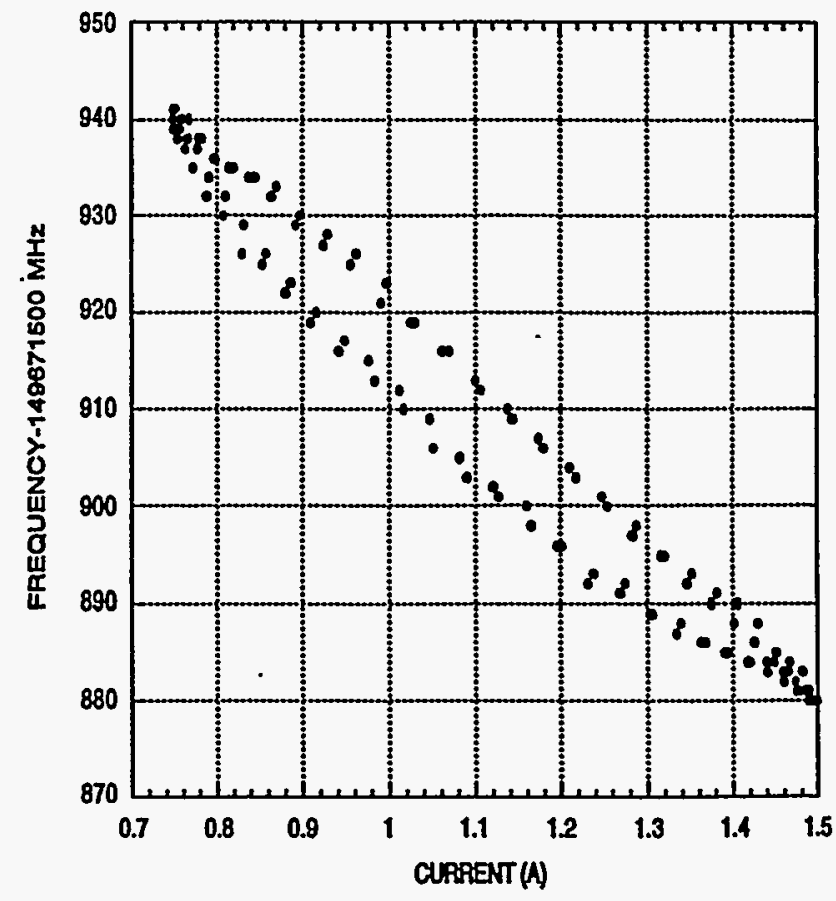

Figure 3. Two solenoid current cycles, $0.05 \mathrm{~Hz}$ sine wave into solenoid. Hysteresis width is $-10 \mathrm{~Hz}$, acceptable for accelerator operation. 
Figure 4 shows all data taken over a one hour period, with a digitally controlled, $0-5 \mathrm{~A}$ power supply. Several cycles of the range in figure 3 are imbedded therein. The logarithmic fit is not quite as good as in figure 2, but still more than adequate for control purposes in a feedback loop. Data from figure 2 is plotted with the triangles for comparison. The $40 \mathrm{~Hz}$ offset is not understood. Helium pressure was not monitored during the test, and could be the cause.

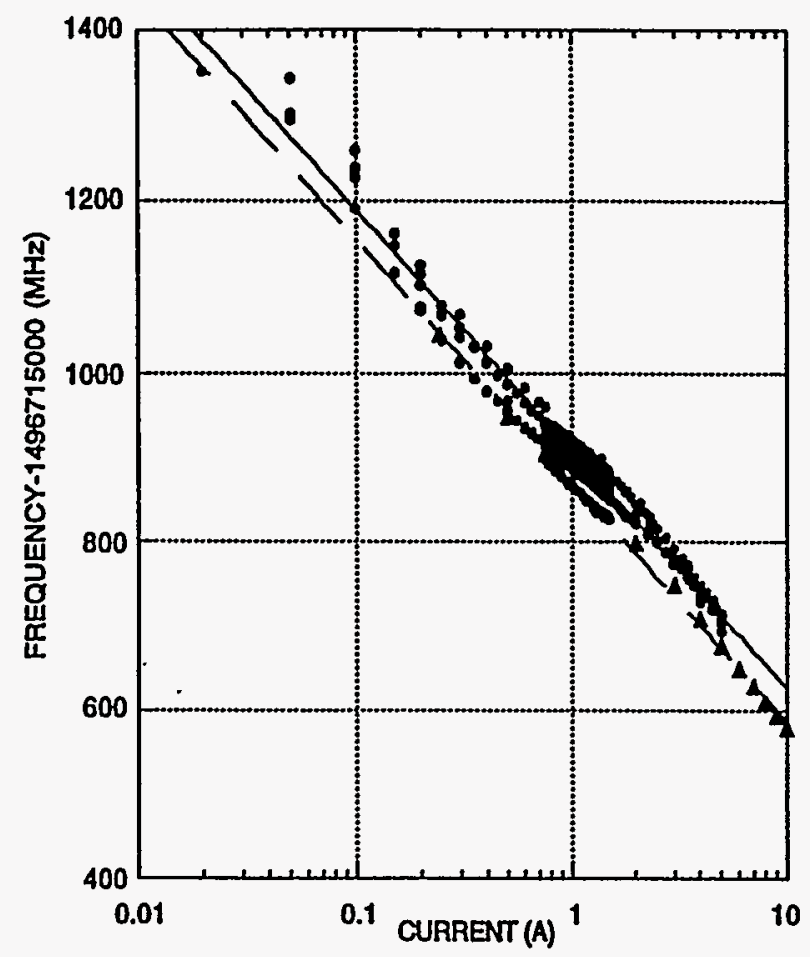

Figure 4. Frequency change as a function of current over one hour, with several cycles $0.75-1.5 \mathrm{~A}$ within sequence.

\section{CONCLUSIONS}

A simple, iron-shielded solenoid was combined with a minor modification to the CEBA mechanical tuner and three supplemental, sheet metal, magnetic shields to form a magnetostrictive tuner. This configuration provides a frequency range of $\pm 300 \mathrm{~Hz}$, with frequency shift proportional to the log of the current in the solenoid. Time response of the tuner is better than one second. Hysteresis due to the nickel magnetostrictive element will not affect accelerator operation for frequency changes within $\pm 50 \mathrm{~Hz}$ and is easily dealt with for the full range available. Two cavities with these tuners are now being assembled into a cryostat and will be tested with up to $5 \mathrm{~mA}$ electron beam within six months. If these tests are successful, the eight cavities in the cryomodule for the Jefferson Lab IR FEL will also be so equipped.

The decision to change the CEBA standard configuration to one with magnetostrictive tuners as cyromodules are reworked will probably await commissioning tests of the IR FEL in 1998. In production quantities, the tuners and their associated electronics add $\$ 40 \mathrm{~K}$ to the cost of each cryomodule; there are 41 cryomodules in the CEBA.

\section{REFERENCES}

[1] H. Grunder, Proceeding of the 1988 Linear Accelerator Conference, and CEBAF report 89-001.

[2] CEBAF Tech Note \#96-026, "Engineering Design Specifications for the IR FEL Driver Transport System" [3] E. Fawcett and G.K. White, Joumal of Applied Physics vol. 38, pp. $1320 \mathrm{ff}$, March 1, 1967 